\title{
Microscopy-Based Approaches to Characterizing Analogs of Classical Electrons in Colloidal Crystals Engineered with DNA
}

\author{
Jingshan Du, Shunzhi Wang, Vinayak Dravid and Chad Mirkin
}

Northwestern University, Evanston, Illinois, United States

Interactions among electrons and atoms are a central premise in physics and chemistry that lead to the formation of many types of molecules and materials. In the science of colloidal crystals, particles are often analogized with atoms, but a particle analog to electrons has not been invoked [1]. This is despite the fact that hundreds of crystal structures with diverse symmetries, lattice parameters, and crystal habits have been designed and synthesized when programmable ligands, specifically DNA, are used as bonds between particles [2,3]. In these systems, DNA-modified nanoparticles (NPs) behave as programmable atom equivalents (PAEs), and their crystallization is governed by a set of design rules and the complementary contact model (CCM) [2], inspired in part by Pauling's rules for "ionic" compounds.

Herein, we report that when reduced in size and DNA grafting density, PAEs behave as electron equivalents (EEs), roaming through and stabilizing the lattices defined by larger PAEs, as electrons do in metals in classical models (Fig. 1A). This topic has been explored both experimentally and computationally [4], but this presentation will focus primarily on the former. In a typical set of experiments, 10-nm-diameter Au NPs were densely functionalized with single-stranded DNA and then hybridized with complementary linker strands to form a rigid duplex region that exposes a non-selfcomplementary, single-stranded overhang. Alone these particles do not form crystals as they are almost solely repulsive. As such, a second set of Au NPs of varying diameter (10, 5, 2, and $1.4 \mathrm{~nm}$ ) were similarly functionalized to contain a complementary DNA overhang. When the two set of particles were mixed in solution, the $10+10,10+5$, and $10+2 \mathrm{~nm}$ combinations form the expected "ionic" CsCl lattice based upon the conventional CCM model, and this was confirmed via both small-angle X-ray scattering (SAXS) and cross-sectional low-angle annular dark field (LAADF) imaging of silica-encased, fixed samples (Fig. 1B and C). However, when 1.4-nm particles were used, SAXS reveals that the structure possesses a bodycentered cubic (bcc) lattice with a markedly decreased lattice parameter. Additionally, cross-sectional LAADF reveals that all bcc lattice sites are occupied by the 10-nm particles, while the 1.4-nm particles are randomly distributed throughout the lattice and act as EEs (Fig. 1C, bottom panel).

To determine the position of the EEs in the bcc lattices formed by the PAEs with minimum perturbation to the structure, we performed cryogenic transmission electron microscopy (cryo-TEM) and then stacked the repeating "unit cell" images along the [111] zone axis (Fig. 1D). By plotting the location of the EEs and calculating their local densities, we found that the large particles assume a bcc lattice and the small particles are randomized throughout that lattice, making it "metallic" (Fig. 1E, left panel). Interestingly, the lattice can be engineered into an "insulating" state by decreasing the input ratio of EEs to PAEs and increasing the DNA linker loading on the EEs. This results in the EEs clustering at positions where they have lower free energy (Fig. 1E, right panel).

In addition to their randomized spatial distribution at a given time, EEs are mobile and can macroscopically diffuse throughout the lattice. To show that EEs can directionally flow in response to a chemical potential difference, "metallic" PAE-EE assemblies were prepared with EEs labeled with Cy5 dye. When incubated in a solution of Cy3-labeled EEs, time-series UV-vis extinction spectroscopy reveal that the Cy5-labeled EEs diffuse into the solution, and the Cy3-labeled EEs diffuse from the solution into 
the crystal (Fig. 1F, upper panel). In contrast, no appreciable exchange is observed for the "ionic" PAEPAE assemblies (Fig. 1F, lower panel). In both cases, the PAEs retain their crystalline structure.

In addition to the bcc structures above, various types of crystal lattices are accessible using the PAE-EE system. By adjusting the input EE:PAE ratio in solution and the total DNA density on the EEs, we synthesized analogs of all three tungsten phases that are known to exist [5]: bcc, fcc, and Frank-Kasper A15. When the input EE:PAE ratio was progressively increased from the equilibrium conditions of the bcc structure, an fcc lattice emerged (Fig. 1G). When both the total DNA coverage on EEs and the input EE:PAE ratio in solution were increased, the A15 phase formed, as evidenced by SAXS (Fig. 1H) and cryo-TEM (Fig. 1I).

Structural complexity can be increased even more by mixing two different sizes of PAEs with EEs functionalized with complementary DNA (Fig. 2A) [6]. This results in a series of "alloy" structures and enables the derivation of a new set of design principles inspired by the Hume-Rothery rules [6]. SAXS and cross-sectional images [LAADF or bright field $(\mathrm{BF})]$ show that when the hydrodynamic radius ratio of the two PAEs decreases from 1, the resulting crystals possess a substitutional, then phase-separated, and then interstitial alloy structure (Fig. 2B-E). To further access the intermetallic structure, 15 hexa(ethylene glycol) spacers were attached to the DNA on $10+40 \mathrm{~nm}$ PAEs with similar hydrodynamic radius (Fig. 2F). This increases the flexibility of the DNA shell for overcoming the strain penalty and resulted in the formation of a CsCl-type intermetallic structure (Fig. 2G and $\mathrm{H}$ ).

Finally, different types of microstructures, including amorphous structures, matrix/precipitates, separate crystals, and core-shell structures can be accessed by controlling the cooling speed during "alloy" annealing, similar to the process used in metallurgy (Fig. 2I-K). Taken together, this work makes the case for describing certain classes of colloidal crystals in a fundamentally new way and opens avenues for accessing new types of structures through colloidal crystal engineering with DNA [8].

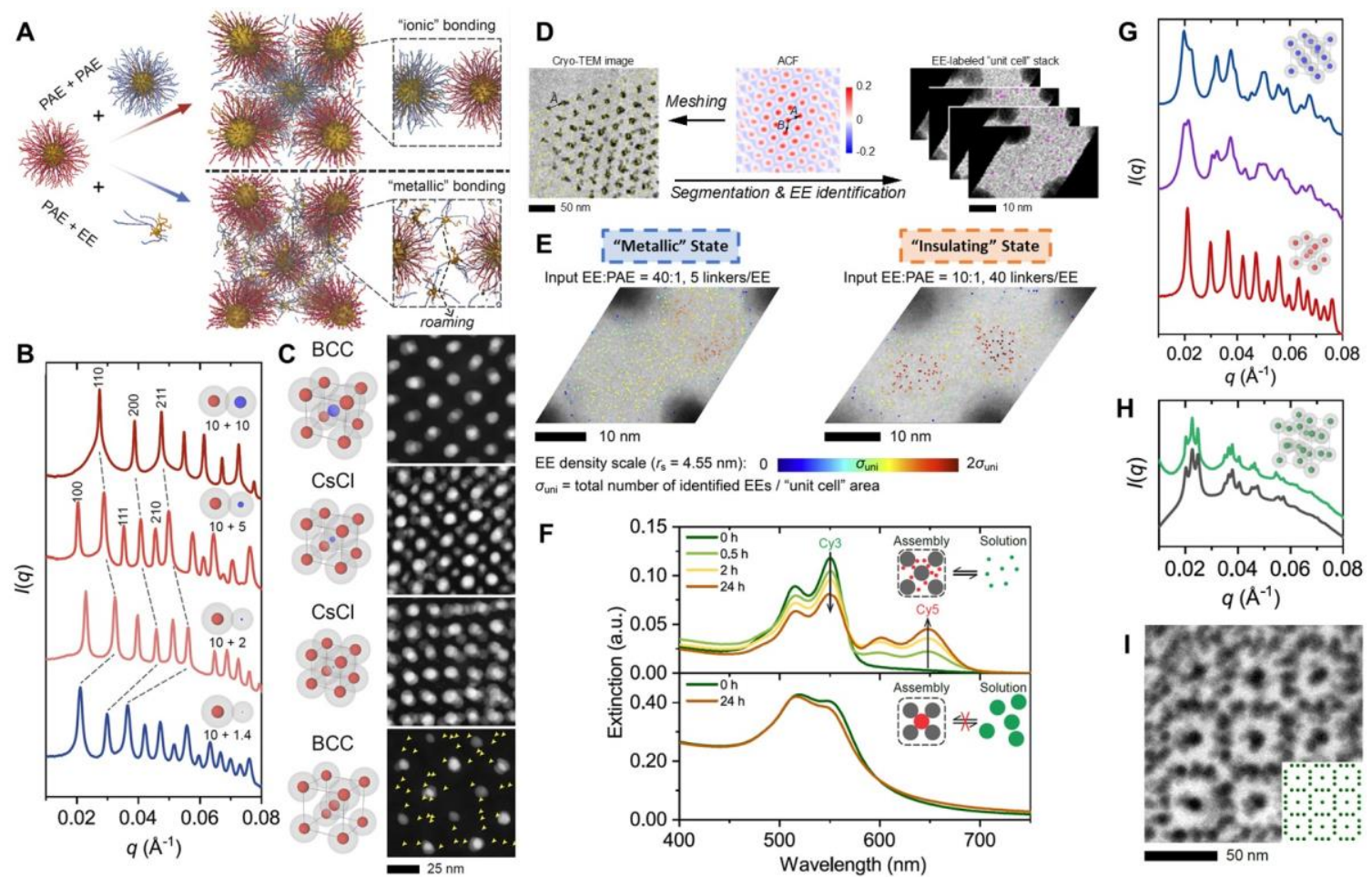


Figure 1. Transition from analogs of atoms to classical electrons. (A) Visualization of the conventional "ionic" colloidal crystals formed by PAEs and the "metallic" colloidal crystals formed by mixing PAEs and EEs. Red and blue strands represent complementary DNA sequences functionalized on Au NPs (yellow balls). (B, C) Four crystalline lattices assembled from 10-nm PAEs and complementary DNAfunctionalized Au NPs (nominal core diameters of 10, 5, 2, and $1.4 \mathrm{~nm}$, respectively): SAXS spectra (B), models of the unit cell, and cross-sectional LAADF images (C) for silica-encapsulated samples. The 1.4$\mathrm{nm}$ Au NPs in (C) (yellow arrows indicate visually identified ones) are dispersed randomly in the lattice and do not occupy specific lattice sites. (D) Workflow for obtaining EE location-labeled "unit cell" images from cryo-TEM using image segmentation. ACF: auto-correlation function. (E) Overlay of averagedintensity TEM images and identified EE locations in "unit cells" along the [111] direction in two states. The formation of the different states is controlled by the input EE:PAE ratio and linker loading on EEs: a "metallic" state where EEs are randomized throughout the lattice, or a "insulating" state where EEs aggregate as local clusters. (F) The exchange of dye-labeled particles between crystalline lattices and solution was monitored by UV-vis spectroscopy. Cy5-DNA-labeled EEs were exchanged from "metallic" PAE-EE assemblies $(10+1.4 \mathrm{~nm}$, bcc) by Cy3-DNA-labeled EEs in the supernatant over $24 \mathrm{~h}$ (upper panel), whereas no appreciable exchange was observed for "ionic" PAE-PAE assemblies $(10+10 \mathrm{~nm}$, bcc, lower panel). (G) SAXS spectra showing the equilibrium phase transition from bcc (red) to a bcc/fcc mixture (purple) and then to a majority fcc phase (blue) as the input EE:PAE ratio increases. $(\mathrm{H})$ Experimental (green) and simulated (black) SAXS spectra of Frank-Kasper (F-K) A15 assemblies formed from PAEs and EEs. (I) Cryo-TEM image of a F-K A15 assembly. Inset: F-K A15 lattice model along the [001] direction. Adapted from ref. [4] with permission, American Association for the Advancement of Science.

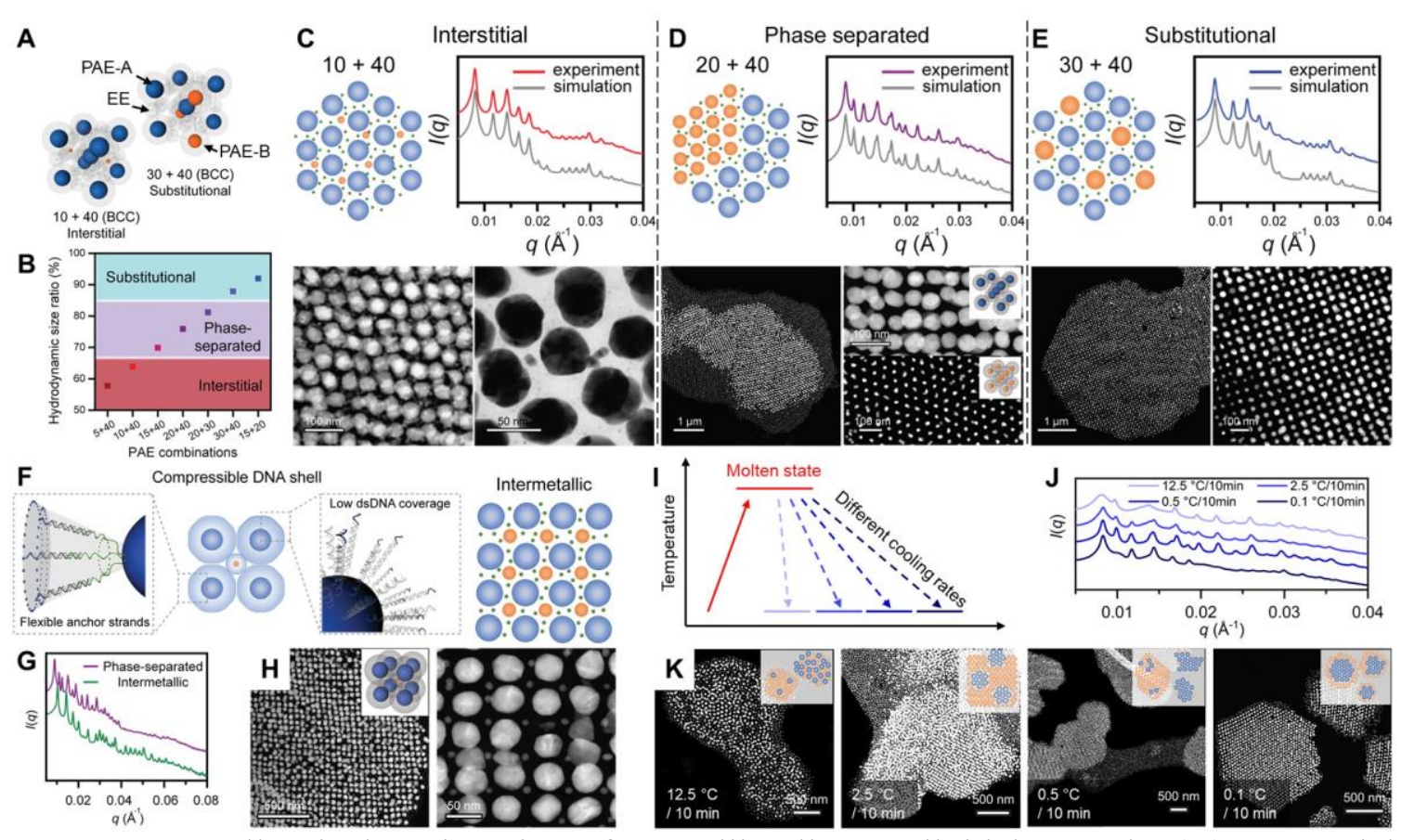

Figure 2. Metallurgical engineering of "metallic alloy" colloidal crystals. (A) 3D models of interstitial $(10+40 \mathrm{~nm})$ and substitutional $(30+40 \mathrm{~nm})$ alloys formed when 2 types of PAEs with different sizes were assembled with EEs. (B) Phase behavior plotted as a function of PAE hydrodynamic radius ratio. The phase structures are determined by SAXS. (C-E) Schematic, SAXS spectra (top), and cross-sectional images (LAADF or BF, bottom) of different types of colloidal alloys: interstitial (C), phase-separated (D), and substitutional (E). The DNA shell on the PAEs and EEs are omitted in the cartoons for clarity. (F) 
Engineering the DNA with hexa-ethylene glycol spacer units (green lines) results in compressible DNA shells which can accommodate local stress induced by lattice mismatch. (G) SAXS reveals the formation of a CsCl-type intermetallic structure when 15 PEG spacers were inserted in the DNA on $10+40 \mathrm{~nm}$ PAEs, and phase-separated structures formed when 6 spacers were used. $(\mathrm{H})$ Cross-sectional LAADF images of the intermetallic structure. (I) After heating to the molten state, the cooling rate controls the microstructure of the colloidal crystals. ( $\mathrm{J}, \mathrm{K})$ Characterization of the kinetic products obtained by four different cooling rates via SAXS (J) and cross-sectional LAADF imaging (K). Inset schematics depict the four types of phase-separated colloidal crystal alloys formed by cooling at different rates. Adapted from ref. [6] with permission, American Chemical Society.

\section{References}

[1] Boles, M. A.; Engel, M.; Talapin, D. V., Chem. Rev. 2016, 116, 11220-11289.

[2] Macfarlane, R. J.; Lee, B.; Jones, M. R.; Harris, N.; Schatz, G. C.; Mirkin, C. A., Science 2011, 334, 204-208.

[3] Auyeung, E.; Li, T. I. N. G.; Senesi, A. J.; Schmucker, A. L.; Pals, B. C.; de la Cruz, M. O.; Mirkin, C. A., Nature 2014, 505, 73-77.

[4] Girard, M.; Wang, S.; Du, J. S.; Das, A.; Huang, Z.; Dravid, V. P.; Lee, B.; Mirkin, C. A.; Olvera de la Cruz, M., Science 2019, 364, 1174-1178.

[5] Lassner, E.; Schubert, W.-D., The Element Tungsten. In Tungsten: Properties, Chemistry, Technology of the Element, Alloys, and Chemical Compounds, Springer US: Boston, MA, 1999; pp 1-59.

[6] Wang, S.; Du, J. S.; Diercks, N. J.; Zhou, W.; Roth, E. W.; Dravid, V. P.; Mirkin, C. A., J. Am. Chem. Soc. 2019, 141, 20443-20450.

[7] Hume-Rothery, W.; Mabbott, G. W.; Evans, K. M. C. Philos. Trans. Royal Soc. B 1934, 233, 1-97.

[8] We gratefully acknowledge the contributions of Martin Girard and Monica Olvera de la Cruz (Northwestern University) whose work with molecular dynamics simulation (not presented) were important for establishing some of the experimental concepts described herein, and Eric W. Roth (Northwestern University) for ultramicrotomy. The authors acknowledge funding from the Air Force Office of Scientific Research (FA9550-17-1-0348), the Vannevar Bush Faculty Fellowship program (N00014-15-1-0043), and the Sherman Fairchild Foundation, Inc. 